\title{
Triplet versus doublet neoadjuvant chemotherapy regimens for locally advanced gastric cancer: a propensity score matching analysis
}

Yonghe Chen ${ }^{1,2 \dagger}$, Jiasheng He ${ }^{1,2 \dagger}$, Dan Liu ${ }^{3 \dagger}$, Jian Xiao ${ }^{4}$, Xijie Chen ${ }^{1,2}$, Haijie Tang ${ }^{4,2}$, Dandong Luo ${ }^{1,2}$, Chenyu Shang ${ }^{3}$, Lei Lian ${ }^{1,2^{*}}$ and Junsheng Peng ${ }^{1,2^{*}}$

\begin{abstract}
Background: To investigate the differences between doublet and triplet neoadjuvant chemotherapy (NAC) regimens in efficacy and safety profile.

Methods: A total of 227 locally advanced gastric cancer (LAGC) patients who received NAC and sequential radical gastrectomy were reviewed. After propensity score matching (PSM), 140 patients with similar baseline characteristics were selected. Among them, 70 received doublet NAC regimens consisted of platinum and fluorouracil; the other 70 received triplet NAC regimens consisted of docetaxel, platinum, and fluorouracil.

Results: The efficacy of doublet and triplet regimens was comparable after propensity score matching in terms of tumor regression (pathological complete response, Doublet 11.4\% vs. Triplet 15.7\%, $p=0.642$ ), achieving of R0 resection (Doublet $88.6 \%$ vs. Triplet $88.6 \%, p=1$ ), 1-year disease-free survival (DFS) (Doublet $77.1 \%$ vs. Triplet 68.6\%, $p=0.178$ ), 3 -years overall survival (OS) (Doublet $54.3 \%$ vs. Triplet $60.9 \%, p=0.941$ ). Post-surgery complications were more common in the triplet cohort (Doublet $5.7 \%$ vs. Triplet $27.1 \%, p=0.001$ ), especially abdominal infection (Doublet $0 \%$ vs. Triplet $11.1 \%, p=0.001$ ).
\end{abstract}

Conclusions: A more intense preoperative triplet NAC regimen does not bring extra downstage effect and survival benefit compared to a doublet regimen. It may even result in a higher risk of post-surgery complications.

Keywords: Gastric cancer, Neoadjuvant chemotherapy, Survival, Propensity score matching

\section{Background}

Gastric cancer is the fifth most common malignancy globally and the third leading cause of cancer-related death [1]. Most patients are diagnosed at the advanced stages of the disease, with an extremely poor prognosis $[2,3]$. For operable locally advanced gastric cancer

\footnotetext{
*Correspondence: lianlei2@mail.sysu.edu.cn; pengjsh@mail.sysu.edu.cn ${ }^{\dagger}$ Yonghe Chen, Jiasheng He and Dan Liu contributed equally to this work. 1 Department of Gastric Surgery, The Sixth Affiliated Hospital, Sun Yat-sen University, 26 Yuancun Erheng Road, Guangzhou 510655, China Full list of author information is available at the end of the article
}

(LAGC), neoadjuvant chemotherapy (NAC) is recommended. However, the recommended regimens vary vastly in different guidelines [4-7]. Despite the variety of recommendations, all recommended regimens fall into two categories: the doublet regimens and the triplet regimens. Generally, the Asian guidelines endorse doublet regimens that combine platinum with fluorouracil or oral fluorouracil derivant (capecitabine or S-1). The other guidelines, such as the European Society for Medical Oncology (ESMO) and the National Comprehensive Cancer Network guideline (NCCN), recommend triplet 
regimens that combine docetaxel, platinum, and fluorouracil, such as the FLOT and the DCF regimen. In recent years, the FLOT regimen has become worldwide popular after the FLOT4 trial demonstrated its superiority over the classic ECF regimen [8]. Researchers proposed that adding docetaxel would significantly improve tumor downstaging, R0 resection rate, and survival [9, 10]. While some other researchers questioned that the addition of docetaxel only increases the toxicity, and its superiority over the modernized doublet regimen such as SOX or CAPOX, is yet to be proven [11]. Till now, a direct comparison between doublet and triplet NAC regimens for LAGC is lacking.

Hence, in this study, we aimed to determine the efficacy and toxicity of doublet and triplet regimens by adding real-world evidence of neoadjuvant chemotherapy for LAGC. We reviewed 227 cases of LAGC patients who received NAC and sequential resection surgery. Their tumor regression grade (TRG), R0 resection rate, toxicity, post-surgery complications and survival were retrospectively compared. We believe the results yielded from this study could provide valuable information for oncologists in choosing treatment strategies for LAGC patients.

\section{Methods}

\section{Study design, inclusion, and exclusion criteria}

This retrospective cohort study aimed to compare the efficacy and safety of doublet and triplet NAC regimens for LAGC patients. All the clinical data were retrieved from the Gastric Cancer Database of The Sixth Affiliated Hospital, Sun Yat-sen University (Guangzhou, China). All patients were followed up via re-examinations in the outpatient clinic and by telephone until mortality due to any reasons or loss of follow-up.

The inclusion criteria were as follows: (i) age between 18 and 80 years old with any gender; (ii) histological diagnosis of gastric/esophagogastric junction adenocarcinoma; (iii) received NAC and sequential radical gastrectomy; (iv) a clinical stage of T2-4N1-3M0; (v) Eastern Cooperative Oncology Group (ECOG) score 0-1.

The exclusion criteria were as follows: (i) received concurrent neoadjuvant radiotherapy or target therapy; (ii) less than 12 months of follow-up; (iii) insufficient staging information or uncertainty of distance metastasis; (iv) secondary malignant tumor.

\section{Pre-intervention staging, neoadjuvant chemotherapy regimen, and surgery}

Before starting the treatments, all patients received enhanced thoracic-abdominal-pelvic computed tomography (CT) scan and/or endoscopic ultrasonography. All the clinical data were reviewed by the gastric cancer multi-disciplinary team consisting of surgeons, oncologists and radiologists. The recommendations of NAC were based on guidelines and the patients' will, the NAC regimen was selected according to the recommendation of guidelines and the physicians' preference. In this study, all the CT image sets were retrieved and re-assessed according to the 8th AJCC staging manual [12].

Triplet NAC regimens used in this study included FLOT and DCF. Doublet regimens used in this study included SOX, CAPOX, and FOLFOX. The details and dose intensity of the regimens are depicted below:

(1) FLOT: docetaxel $50 \sim 60 \mathrm{mg} / \mathrm{m}^{2}$, oxaliplatin $85 \mathrm{mg} /$ $\mathrm{m}^{2}$, and fluorouracil $2800 \mathrm{mg} / \mathrm{m}^{2}$; every 2 weeks;

(2) DCF: docetaxel $50 \mathrm{mg} / \mathrm{m}^{2}$, cisplatin $50 \mathrm{mg} / \mathrm{m}^{2}$, and fluorouracil $2800 \mathrm{mg} / \mathrm{m}^{2}$; every 2 weeks;

(3) SOX: oxaliplatin $130 \mathrm{mg} / \mathrm{m}^{2}$, tegafur gimeracil oteracil potassium capsule $40-60 \mathrm{mg}$ bid Day1Day14; every 3 weeks;

(4) CAPOX: oxaliplatin $130 \mathrm{mg} / \mathrm{m}^{2}$, capecitabine $1000 \mathrm{mg} / \mathrm{m}^{2}$ bid Day1-Day14; every 3 weeks;

(5) FOLFOX: oxaliplatin $85 \mathrm{mg} / \mathrm{m}^{2}$, fluorouracil $2800 \mathrm{mg} / \mathrm{m}^{2}$; every 2 weeks.

After the completion of NAC, the resectability of the primary tumor was confirmed by the multi-disciplinary team. All patients enrolled received curative tumor resection (total or subtotal gastrectomy) with standard D2 lymphadenectomy. A throughout examination of the abdominal cavity was routinely performed to determine the status of peritoneum metastasis before the resection.

\section{Pathological response, toxicity, and post-surgery complications evaluation}

All resected specimens were examined to determine pathological stages and histological response to NAC. Tumor regression grades (TRG) were determined by the number of viable tumor cells that remained in the tumor, according to the Ryan standard [13, 14]. Grade 0 (complete response): no tumor cells remained; Grade 1 (major response): scattered single tumor cells remained; Grade 2 (moderate response): clustered tumor cells remained with fibrosis; Grade 3 (minor response): extensive tumor cells remained. Neoadjuvant chemotherapy related toxicity was evaluated according to the Common Terminology Criteria for Adverse Events version 5.0 [15]. Post-surgery complications were graded according to the ClavienDindo classification system [16]. Grade 2-4 complications, which mean complications that required medical or surgical interventions, were recorded. 


\section{Follow-up}

Following completion of the treatment, follow-up studies were conducted once every two months in the first six months and then once every three months, subsequently. Each follow-up study included medical history, physical examination, routine blood tests, comprehensive biochemical examinations, thoracic-abdominal-pelvic CT scan, and superficial lymph node B-ultrasonography.

\section{Propensity score matching}

A propensity score matching (PSM) method was used for the patients enrolled in this study to select matching pairs with similar baseline characteristics in the two cohorts [17]. The matching factors were sex, age, tumor location, differentiation, diabetes, clinical $\mathrm{T} / \mathrm{N}$ stage, and clinical stage groupings. The matching ratio was $1: 1$, and the caliper was 0.01 . The matched pairs were divided into the doublet cohort and the triplet cohort.

\section{Data analysis}

The normality of data was assessed using the Kolmogorov-Smirnov test and normal probability plots. Parameters that were not normally distributed were expressed in the median (upper quartile to lower quartile) and were analyzed using non-parametric tests: Mann-Whitney test or Kruskal-Wallis test, as appropriate. Normally distributed parameters were expressed in the form of mean \pm standard deviation and were analyzed by Student's $t$-test. Categorical variables were analyzed by the chi-square test. The survival difference was compared using the Kaplan-Meier method, and the hazard ratios were calculated in the Cox regression model. A $p$-value $<0.05$ was identified as statistically significant. All statistical analyses were performed using the SPSS software version 25.0 (IBM, Armonk, NY, USA) and the R software version 4.0.2 (The R Foundation for Statistical Computing, Vienna, Austria; www.r-project.org).

\section{Results}

\section{Patients characteristics}

From February 2013 to December 2018, 265 eligible patients who received neoadjuvant chemotherapy and D2 radical gastrectomy were identified. As depicted in Fig. 1, 227 patients were included in the study after the screening, of which 91 received doublet regimen and 136 received triplet regimen for NAC. After propensity score matching, 140 patients with locally advanced lesions and similar characteristics were selected, with 70 patients in each cohort.

As shown in Table 1, baseline characteristics in the two study cohorts were similar. Patients were mostly male, with a median age of 60 years, and the tumor mainly was poorly differentiated adenocarcinoma. However, before PSM, patients in the triplet cohort were in significantly more advanced clinical stages, especially the $\mathrm{cN}$ stage. After PSM, both Clinical $\mathrm{T}$ and $\mathrm{N}$ stages were almost identical in both cohorts.

\section{Neoadjuvant chemotherapy and toxicity}

The FLOT and SOX were the mainstream regimens in our study, which took up $90 \%$ of the total sample. Other regimens included the FOLFOX, CAPOX, and the DCF regimen, all are commonly used regimens in clinical practice. All patients received a median of 4 cycles of NAC before surgery. The toxicity profiles were depicted in Table 2. Before and after PSM, the triplet cohort had a higher incidence rate of neutropenia and anemia, while thrombocytopenia was more common in the doublet cohort. The incidence rate of grade $3 / 4$ hematological toxicity was not significantly different.

\section{Surgery, pathological findings, and complications}

As depicted in Table 3, no perceptible differences were observed in terms of the R0 resection rate between the two groups (Doublet $90.1 \%$, 82/91 vs. Triplet $88.2 \%$, $120 / 136, p=0.829)$. After PSM analysis, the R0 resection rate was similar in the doublet group and the triplet cohort (both 88.6\%, 62/70, $p=0.1$ ). The incidence rate of complications (Clavien-Dindo grade 2-4) was significantly higher in the triplet cohort (before PSM: Doublet $8.8 \%, 8 / 91$ vs. Triplet $27.2 \%, 37 / 136, p=0.001$; after PSM: Doublet $5.7 \%, 4 / 70$ vs. Triplet $27.1 \%, 19 / 70, \mathrm{p}=0.001$ ), especially surgery-related abdominal infections, caused mainly by anastomotic leakage. As for pathological findings, TRG in the two cohorts was statistically similar whether it was before or after the PSM analysis. The pathological complete response (grade 0: before PSM, Doublet $11.0 \%, 10 / 91$ vs. Triplet $16.2 \%, 22 / 136, p=0.686$; after PSM, Doublet $11.4 \%, 8 / 70$ vs. Triplet $15.7 \%, 11 / 70$, $p=0.642$ ) was higher in the triplet cohort, but the difference was insignificant. Others were similar, including the post-surgical ypTN stages spectrum and numbers of positive/total harvested lymph nodes.

\section{Survival analysis}

The median follow-up time was 31 months. As shown in Fig. 2, before PSM, the disease-free survival (DFS) in the triplet cohort was shorter, but the difference becomes insignificant after PSM (1-year DFS rate, Doublet 77.1\% vs. Triplet $68.6 \%, p=0.178$ ). The overall survival (OS) was similar in both cohorts, before or after PSM (3-years OS rate after PSM, Doublet $54.3 \%$ vs. Triplet $60.9 \%$, $p=0.941$ ). In subgroup survival analysis (Fig. 3 ), the triplet cohort also failed to exhibit any superiority in any subgroups. In the subgroup of patients with moderately 


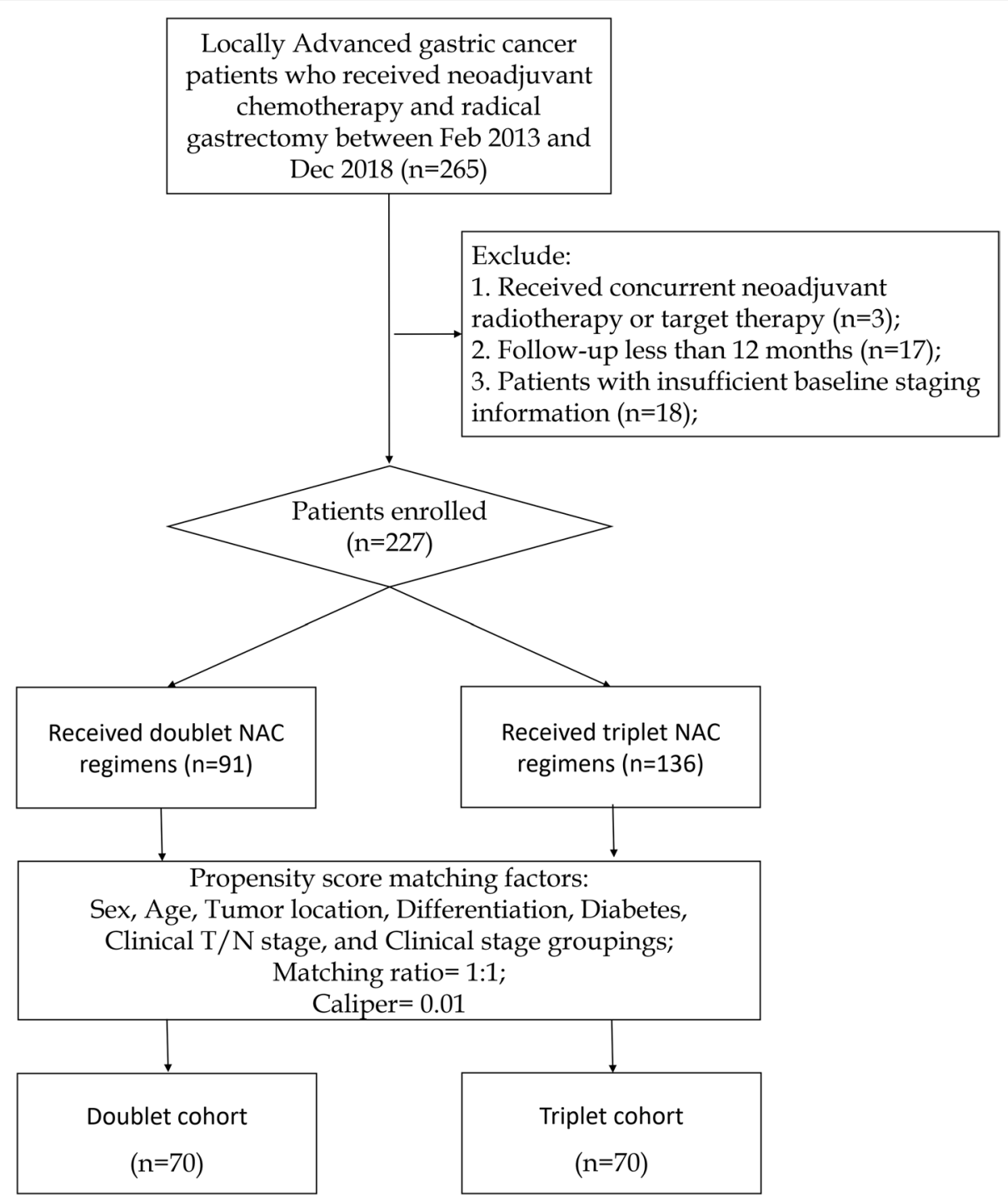

Fig. 1 The flowchart showing the process of patients' enrollment and propensity score matching

differentiated adenocarcinoma, triplet NAC was even correlated with shorten DFS. Therefore, these data suggested that the triplet regimen has brought no additional survival benefit compared with the doublet regimen.

\section{Discussion}

The triplet regimens, such as ECF/DCF/FLOT, have been endorsed as NAC regimens for LAGC by many reputable gastric cancer guidelines, such as the NCCN/ESMO. In 2006, the MAGIC trial [18] proved that NAC with epirubicin, cisplatin and fluorouracil (the ECF regimen) improved survival compared with surgery alone, setting ECF as the first recommended NAC regimen. However, many studies have reported that ECF was less tolerable in clinical practice due to the high toxicity profile, limiting its use. Later on, in the FLOT4 [8] study, a triplet regimen consisted of docetaxel, oxaliplatin and fluorouracil (the FLOT regimen) exhibited a more satisfactory efficacy than the ECF regimen, reaching a median survival of 50 months, making it the standard NAC regimen ever since. However, doublet regimens combining oxaliplatin and fluorouracil, such as SOX or CAPOX, are still commonly used as NAC regimens in Asian countries, including China, Japan, and Korea [19-21]. Some preliminary randomized trials showed that these modernized doublet regimens also exhibit satisfactory efficacy $[22,23]$. Thus some researchers proposed that these doublet regimens could also be used as first-line NAC regimen, with efficacy that is non-inferior to triplet regimen and lower toxicity profiles. While some insisted that triplet regimens 
Table 1 Patients' characteristics before and after propensity score matching (PSM)

\begin{tabular}{|c|c|c|c|c|c|c|c|c|}
\hline \multirow[t]{2}{*}{ Characteristic } & \multicolumn{4}{|l|}{ Before PSM } & \multicolumn{4}{|l|}{ After PSM } \\
\hline & $\begin{array}{l}\text { All } \\
n=227(\%)\end{array}$ & $\begin{array}{l}\text { Double-agent } \\
n=91(\%)\end{array}$ & $\begin{array}{l}\text { Triple-agent } \\
n=136(\%)\end{array}$ & $p$-value & $\begin{array}{l}\text { All } \\
n=140(\%)\end{array}$ & $\begin{array}{l}\text { Double-agent } \\
n=70(\%)\end{array}$ & $\begin{array}{l}\text { Triple-agent } \\
n=70(\%)\end{array}$ & $p$-value \\
\hline \multicolumn{9}{|l|}{ Sex } \\
\hline Male & $173(76.2)$ & $68(74.7)$ & $105(77.2)$ & 0.786 & $107(76.4)$ & $53(75.7)$ & $54(77.1)$ & 1 \\
\hline Female & $54(23.8)$ & $23(25.3)$ & $31(22.8)$ & & $33(23.6)$ & $17(24.3)$ & $16(22.9)$ & \\
\hline Age & $59[50,64]$ & $60[50,64]$ & $59[49,64]$ & 0.398 & $59[49,64.25]$ & $59.5[52.25,64]$ & $56[48.25,66]$ & 0.347 \\
\hline$\leq 60$ years & $124(93.8)$ & $48(92.3)$ & $76(94.9)$ & 0.684 & 78 (55.7) & $38(54.3)$ & $40(57.1)$ & 0.865 \\
\hline$>60$ years & $103(6.2)$ & $43(7.7)$ & $60(5.1)$ & & $62(44.3)$ & $32(45.7)$ & $30(42.9)$ & \\
\hline \multicolumn{9}{|c|}{ Diabetes mellitus } \\
\hline Yes & $16(7)$ & $5(5.5)$ & $11(8.1)$ & 0.485 & $12(7.9)$ & $5(6.7)$ & $7(9.1)$ & 0.57 \\
\hline No & $211(93)$ & $86(94.5)$ & $125(91.9)$ & & $140(92.1)$ & $70(93.3)$ & $70(90.9)$ & \\
\hline \multicolumn{9}{|l|}{ Location } \\
\hline Upper & 87 (38.3) & $28(30.8)$ & $59(43.4)$ & 0.121 & $50(35.7)$ & $25(35.7)$ & $25(35.7)$ & 1 \\
\hline Middle & $49(21.6)$ & $20(22.0)$ & $29(21.3)$ & & $34(24.3)$ & $17(24.3)$ & $17(24.3)$ & \\
\hline Lower & $91(40.1)$ & $43(47.3)$ & $48(35.3)$ & & $56(40.0)$ & $28(40.0)$ & $28(40.0)$ & \\
\hline \multicolumn{9}{|c|}{ Differentiation of adenocarcinoma } \\
\hline Well & $7(3.1)$ & $3(3.3)$ & $4(2.9)$ & 0.981 & - & - & - & 1 \\
\hline Moderately & $66(29.1)$ & $26(28.6)$ & $40(29.4)$ & & $39(27.9)$ & $20(28.6)$ & $19(27.1)$ & \\
\hline Poorly & $154(67.8)$ & $62(68.1)$ & $92(67.6)$ & & $101(72.1)$ & $50(71.4)$ & $51(72.9)$ & \\
\hline \multicolumn{9}{|l|}{ Clinical T stage } \\
\hline T2 & $4(1.8)$ & $2(2.2)$ & $2(1.5)$ & 0.512 & $2(1.4)$ & $1(1.4)$ & $1(1.4)$ & 0.998 \\
\hline T3 & $141(62.1)$ & $61(67.0)$ & $80(58.8)$ & & $87(62.1)$ & $43(61.4)$ & $44(62.9)$ & \\
\hline$T 4 a$ & $55(24.2)$ & $20(22.0)$ & $35(25.7)$ & & $35(25.0)$ & $18(25.7)$ & $17(24.3)$ & \\
\hline$T 4 b$ & $27(11.9)$ & $8(8.8)$ & $19(14.0)$ & & $16(11.4)$ & $8(11.4)$ & $8(11.4)$ & \\
\hline \multicolumn{9}{|l|}{ Clinical N stage } \\
\hline NO & $2(0.9)$ & $2(2.2)$ & $0(0.0)$ & $<0.001$ & - & - & - & 0.983 \\
\hline N1 & $81(35.7)$ & $48(52.7)$ & $33(24.3)$ & & $61(43.6)$ & $31(44.3)$ & $30(42.9)$ & \\
\hline N2 & $97(42.7)$ & $29(31.9)$ & $68(50.0)$ & & $55(39.3)$ & 27 (38.6) & $28(40.0)$ & \\
\hline N3 & $47(20.7)$ & $12(13.2)$ & $35(25.7)$ & & $24(17.1)$ & $12(17.1)$ & $12(17.1)$ & \\
\hline \multicolumn{9}{|c|}{ Clinical stage groupings } \\
\hline$\| A$ & $4(1.8)$ & $2(2.2)$ & $2(1.5)$ & 0.22 & $2(1.4)$ & $1(1.4)$ & $1(1.4)$ & 1 \\
\hline$\| B$ & $2(0.9)$ & $2(2.2)$ & $0(0.0)$ & & - & - & - & \\
\hline III & $194(85.5)$ & 79 (86.8) & $115(84.6)$ & & $122(87.1)$ & $61(87.1)$ & $61(87.1)$ & \\
\hline IVA & $27(11.9)$ & $8(8.8)$ & $19(14.0)$ & & $16(11.4)$ & $8(11.4)$ & $8(11.4)$ & \\
\hline \multicolumn{9}{|l|}{ Regimen } \\
\hline FOLFOX6 & $8(3.5)$ & $8(8.8)$ & - & & $4(3.5)$ & $4(5.7)$ & - & \\
\hline CAPOX & $12(5.3)$ & $12(13.2)$ & - & & $7(5.3)$ & $7(10.0)$ & - & \\
\hline SOX & $71(31.3)$ & $71(78.0)$ & - & - & $59(31.3)$ & $59(84.3)$ & - & - \\
\hline$D C F$ & $5(2.2)$ & - & $5(3.7)$ & & $4(2.2)$ & - & $4(5.7)$ & \\
\hline FLOT & $131(57.7)$ & - & $131(96.3)$ & & $66(57.7)$ & - & $66(94.3)$ & \\
\hline Cycles & $4.00[4.00,4.00]$ & $4.00[3.00,4.00]$ & $4.00[4.00,5.00]$ & $<0.001$ & $4.00[4.00,4.00]$ & $4.00[3.00,4.00]$ & $4.00[4.00,5.00]$ & 0.006 \\
\hline
\end{tabular}

such as FLOT or DCF should be the golden standard because a more intense preoperative chemotherapy could better downstage the tumor, improve $\mathrm{R} 0$ resection rate, and eventually prolong survival. Thus, the issue of which should be the most appropriate NAC regimen for LAGC, doublet or triplet, is still left unsettle.
In this study, we retrospectively reviewed 227 cases of LAGC who received double or triplet NAC to investigate the toxicity profile and efficacy. We use PSM to select two cohorts with similar characteristics, making them comparable. The matching factors were sex, age, tumor location, differentiation, diabetes, clinical $\mathrm{T} / \mathrm{N}$ 
Table 2 Hematological toxicity according to the CTCAE 5.0

\begin{tabular}{|c|c|c|c|c|c|c|c|c|}
\hline \multirow[t]{2}{*}{ Characteristic } & \multicolumn{4}{|l|}{ Before PSM } & \multicolumn{4}{|l|}{ After PSM } \\
\hline & $\begin{array}{l}\text { All } \\
n=227(\%)\end{array}$ & $\begin{array}{l}\text { Double-agent } \\
n=91(\%)\end{array}$ & $\begin{array}{l}\text { Triple-agent } \\
n=136(\%)\end{array}$ & $p$-value & $\begin{array}{l}\text { All } \\
n=140(\%)\end{array}$ & $\begin{array}{l}\text { Double-agent } \\
n=70(\%)\end{array}$ & $\begin{array}{l}\text { Triple-agent } \\
n=70(\%)\end{array}$ & $p$-value \\
\hline $\begin{array}{l}\text { Overall grade } 3 / 4 \\
\text { hematological } \\
\text { toxicity }\end{array}$ & $130(57.3)$ & 47 (51.6) & $83(61.0)$ & 0.206 & 75 (53.6) & $33(47.1)$ & $42(60.0)$ & 0.175 \\
\hline \multicolumn{9}{|l|}{ Anemia } \\
\hline Grade 1 & $35(15.4)$ & $22(24.2)$ & $13(9.6)$ & 0.016 & $18(12.9)$ & $14(20.0)$ & $4(5.7)$ & 0.044 \\
\hline Grade 2 & $81(35.7)$ & $28(30.8)$ & $53(39.0)$ & & $53(37.9)$ & $23(32.9)$ & $30(42.9)$ & \\
\hline Grade 3 & $69(30.4)$ & $23(25.3)$ & $46(33.8)$ & & $45(32.1)$ & 20 (28.6) & 25 (35.7) & \\
\hline Grade 4 & $24(10.6)$ & $8(8.8)$ & $16(11.8)$ & & $11(7.9)$ & $4(5.7)$ & $7(10.0)$ & \\
\hline \multicolumn{9}{|l|}{ Neutropenia } \\
\hline Grade 1 & $30(13.2)$ & $12(13.2)$ & $18(13.2)$ & 0.024 & $20(14.3)$ & $9(12.9)$ & $11(15.7)$ & 0.497 \\
\hline Grade 2 & $68(30.0)$ & $31(34.1)$ & $37(27.2)$ & & $50(35.7)$ & 27 (38.6) & $23(32.9)$ & \\
\hline Grade 3 & $50(22.0)$ & $26(28.6)$ & $24(17.6)$ & & $30(21.4)$ & $16(22.9)$ & $14(20.0)$ & \\
\hline Grade 4 & $30(13.2)$ & $5(5.5)$ & $25(18.4)$ & & $14(10.0)$ & $4(5.7)$ & $10(14.3)$ & \\
\hline \multicolumn{9}{|l|}{ FebrileNeutropenia } \\
\hline Grade 0 & $220(96.9)$ & 90 (98.9) & $130(95.6)$ & 0.306 & $134(95.7)$ & $69(98.6)$ & 65 (92.9) & 0.211 \\
\hline Grade 3 & $7(3.1)$ & $1(1.1)$ & $6(4.4)$ & & $6(4.3)$ & $1(1.4)$ & $5(7.1)$ & \\
\hline \multicolumn{9}{|l|}{ Thrombocytopenia } \\
\hline Grade 1 & $38(16.7)$ & $18(19.8)$ & $20(14.7)$ & $<0.001$ & $24(17.1)$ & $15(21.4)$ & $9(12.9)$ & $<0.001$ \\
\hline Grade 2 & $33(14.5)$ & $27(29.7)$ & $6(4.4)$ & & $25(17.9)$ & $21(30.0)$ & $4(5.7)$ & \\
\hline Grade 3 & $18(7.9)$ & $10(11.0)$ & $8(5.9)$ & & $9(6.4)$ & $6(8.6)$ & $3(4.3)$ & \\
\hline Grade 4 & $4(1.8)$ & $0(0.0)$ & $4(2.9)$ & & $2(1.4)$ & $0(0.0)$ & $2(2.9)$ & \\
\hline \multicolumn{9}{|l|}{ Creatinine elevation } \\
\hline Grade 1 & $24(10.6)$ & $12(13.2)$ & $12(8.8)$ & 0.606 & $17(12.1)$ & $10(14.3)$ & $7(10.0)$ & 0.260 \\
\hline Grade 2 & $7(3.1)$ & $2(2.2)$ & $5(3.7)$ & & $6(4.3)$ & $1(1.4)$ & $5(7.1)$ & \\
\hline Grade 3 & $1(0.4)$ & $0(0.0)$ & $1(0.7)$ & & $1(0.7)$ & $0(0.0)$ & $1(1.4)$ & \\
\hline Grade 4 & $1(0.4)$ & $0(0.0)$ & $1(0.7)$ & & $1(0.7)$ & $0(0.0)$ & $1(1.4)$ & \\
\hline \multicolumn{9}{|c|}{ Alanine transaminase elevation } \\
\hline Grade 1 & $130(57.3)$ & $62(68.1)$ & $68(50.0)$ & 0.007 & $87(62.1)$ & 48 (68.6) & $39(55.7)$ & 0.253 \\
\hline Grade 2 & $20(8.8)$ & $7(7.7)$ & $13(9.6)$ & & $12(8.6)$ & $5(7.1)$ & $7(10.0)$ & \\
\hline Grade 3 & $19(8.4)$ & $5(5.5)$ & $14(10.3)$ & & $10(7.1)$ & $4(5.7)$ & $6(8.6)$ & \\
\hline Grade 4 & $3(1.3)$ & $3(3.3)$ & $0(0.0)$ & & $2(1.4)$ & $2(2.9)$ & $0(0.0)$ & \\
\hline
\end{tabular}

Abbreviations: PSM Propensity score matching; CTCAE Common Terminology Criteria for Adverse Events

stage, and clinical stage groupings, all pre-intervention factors related to the treatment outcome and survival. Since the pathological stage may be altered by the NAC, clinical stages are the most important confounding factor for this study [24]. After PSM, the clinical stages were almost identical in the two cohorts. We found that the triplet NAC regimen is not only no better than the doublet regimen in terms of efficacy, it may also potentially bring more post-surgery complications.

Firstly, the most important indicators for efficacy valuation are the $\mathrm{R} 0$ resection rate, tumor regression grade (TRG), and survival. TRG is the classification of cancer response to preoperative treatment based on the residual cells remained in the tumor lesions. The best case is pathological complete response (PCR), which means no residual cells remained [25]. TRG is considered a more objective indicator of tumor response than radiological response grade since pseudoprogression is possible when evaluating empty organs on radiological images [26]. Our results showed that the triplet regimens did not bring forth a higher PCR rate, nor a better downstaging, than the doublet regimens. The $\mathrm{R} 0$ resection rate was not significantly improved by the triplet regimens either. R0 resection means a more thorough clearance of the tumor and is a major achievement goal of NAC, which is related to prolonged survival. The DFS and OS were not improved in the triplet cohort either. Deteriorated DFS was observed in the subgroup with moderate 
Table 3 Surgical outcomes, pathological findings, and adjuvant chemotherapy

\begin{tabular}{|c|c|c|c|c|c|c|c|c|}
\hline \multirow[t]{2}{*}{ Characteristic } & \multicolumn{4}{|l|}{ Before PSM } & \multicolumn{4}{|l|}{ After PSM } \\
\hline & $\begin{array}{l}\text { All } \\
n=227(\%)\end{array}$ & $\begin{array}{l}\text { Double-agent } \\
n=91(\%)\end{array}$ & $\begin{array}{l}\text { Triple-agent } \\
n=136(\%)\end{array}$ & $p$-value & $\begin{array}{l}\text { All } \\
n=140(\%)\end{array}$ & $\begin{array}{l}\text { Double-agent } \\
n=70(\%)\end{array}$ & $\begin{array}{l}\text { Triple-agent } \\
n=70(\%)\end{array}$ & $p$-value \\
\hline \multicolumn{9}{|l|}{ Laparoscopic } \\
\hline No & $41(18.1)$ & $10(11.0)$ & $31(22.8)$ & \multirow[t]{2}{*}{0.037} & $29(20.7)$ & $10(14.3)$ & $19(27.1)$ & \multirow[t]{2}{*}{0.095} \\
\hline Yes & $186(81.9)$ & $81(89.0)$ & $105(77.2)$ & & $111(79.3)$ & $60(85.7)$ & $51(72.9)$ & \\
\hline \multicolumn{9}{|l|}{ Resection extend } \\
\hline Distal & $93(41.0)$ & $45(49.5)$ & $48(35.3)$ & \multirow[t]{2}{*}{0.047} & $59(42.1)$ & $31(44.3)$ & $28(40.0)$ & \multirow[t]{2}{*}{0.732} \\
\hline Total & $134(59.0)$ & $46(50.5)$ & $88(64.7)$ & & $81(57.9)$ & $39(55.7)$ & $42(60.0)$ & \\
\hline \multicolumn{9}{|l|}{$\mathrm{R} 0$ resection } \\
\hline RO & $202(89.0)$ & $82(90.1)$ & $120(88.2)$ & \multirow[t]{2}{*}{0.829} & 124 (88.6) & 62 (88.6) & $62(88.6)$ & \multirow[t]{2}{*}{1} \\
\hline R1 or R2 & $25(11.0)$ & $9(9.9)$ & $16(11.8)$ & & $16(11.4)$ & $8(11.4)$ & $8(11.4)$ & \\
\hline \multicolumn{9}{|l|}{ Complications $^{\mathrm{a}}$} \\
\hline Overall & $45(19.82)$ & $8(8.8)$ & $37(27.2)$ & 0.001 & $23(16.4)$ & $4(5.7)$ & $19(27.1)$ & 0.001 \\
\hline Abdominal infection & $15(6.6)$ & $1(1.1)$ & $14(10.3)$ & 0.014 & $8(5.7)$ & $0(0.0)$ & $8(11.1)$ & 0.011 \\
\hline Anastomotic leakage & $12(5.3)$ & $1(1.1)$ & $11(8.1)$ & 0.045 & $7(5.0)$ & $0(0.0)$ & $7(10.0)$ & 0.020 \\
\hline Digestive obstruction & $2(0.9)$ & $1(1.1)$ & $1(0.7)$ & 1 & $1(0.7)$ & $1(1.4)$ & $0(0.0)$ & 1 \\
\hline Pneumonia & $2(0.9)$ & $1(1.1)$ & $1(0.7)$ & 1 & $2(1.4)$ & $1(1.4)$ & $1(1.4)$ & 1 \\
\hline Bleeding & $5(2.2)$ & $2(2.2)$ & $3(2.2)$ & 1 & $2(1.4)$ & $1(1.4)$ & $1(1.4)$ & 1 \\
\hline Arrhythmia & $2(0.9)$ & $1(1.1)$ & $1(0.7)$ & 1 & $1(0.7)$ & $0(0.0)$ & $1(1.4)$ & 1 \\
\hline Pleural effusion & $7(3.1)$ & $1(1.1)$ & $6(4.4)$ & 0.306 & $2(1.4)$ & $1(1.4)$ & $1(1.4)$ & 0.612 \\
\hline \multicolumn{9}{|l|}{ Tumor regression grade } \\
\hline Grade O(complete response) & $32(14.1)$ & $10(11.0)$ & $22(16.2)$ & \multirow[t]{4}{*}{0.686} & $19(13.6)$ & $8(11.4)$ & $11(15.7)$ & \multirow[t]{4}{*}{0.642} \\
\hline Grade 1 (major response) & $37(16.3)$ & $14(15.4)$ & $23(16.9)$ & & $24(17.1)$ & $10(14.3)$ & $14(20.0)$ & \\
\hline Grade 2 (moderate response) & $128(56.4)$ & $54(59.3)$ & $74(54.4)$ & & 79 (56.4) & $42(60.0)$ & $37(52.9)$ & \\
\hline Grade 3(minor response) & $30(13.2)$ & $13(14.3)$ & $17(12.5)$ & & $18(12.9)$ & $10(14.3)$ & $8(11.4)$ & \\
\hline \multicolumn{9}{|l|}{ Pathological T stage } \\
\hline TO & $35(15.4)$ & $10(11.0)$ & $25(18.4)$ & \multirow[t]{6}{*}{0.240} & $20(14.3)$ & $8(11.4)$ & $12(17.1)$ & \multirow[t]{6}{*}{0.783} \\
\hline$T 1$ & $25(11.0)$ & $11(12.1)$ & $14(10.3)$ & & $15(10.7)$ & $8(11.4)$ & $7(10.0)$ & \\
\hline T2 & $23(10.1)$ & $13(14.3)$ & $10(7.4)$ & & $17(12.1)$ & $10(14.3)$ & $7(10.0)$ & \\
\hline T3 & $137(60.4)$ & $56(61.5)$ & $81(59.6)$ & & $85(60.7)$ & $43(61.4)$ & $42(60.0)$ & \\
\hline$T 4 a$ & $6(2.6)$ & $1(1.1)$ & $5(3.7)$ & & $3(2.1)$ & $1(1.4)$ & $2(2.9)$ & \\
\hline$T 4 b$ & $1(0.4)$ & $0(0.0)$ & $1(0.7)$ & & - & - & - & \\
\hline \multicolumn{9}{|l|}{ Pathological N stage } \\
\hline NO & $107(47.1)$ & $50(54.9)$ & $57(41.9)$ & \multirow[t]{5}{*}{0.183} & $73(52.1)$ & $40(57.1)$ & $33(47.1)$ & \multirow[t]{5}{*}{0.723} \\
\hline N1 & $48(21.1)$ & $17(18.7)$ & $31(22.8)$ & & $25(17.9)$ & $12(17.1)$ & $13(18.6)$ & \\
\hline N2 & $38(16.7)$ & $12(13.2)$ & $26(19.1)$ & & $21(15.0)$ & $10(14.3)$ & $11(15.7)$ & \\
\hline N3a & $7(3.1)$ & $4(4.4)$ & $3(2.2)$ & & $5(3.6)$ & $2(2.9)$ & $3(4.3)$ & \\
\hline N3b & $18(7.9)$ & $7(7.7)$ & $11(8.1)$ & & $11(7.9)$ & $5(7.1)$ & $6(8.6)$ & \\
\hline Positive lymph nodes & $1[0,4]$ & $0[0,3]$ & $1[0,5]$ & 0.082 & $0[0,4]$ & $0[0,2]$ & $1[0,5]$ & 0.205 \\
\hline Harvested lymph nodes & $28[19,37]$ & $28[22,37.50]$ & $28[19,36.25]$ & 0.793 & $28.5[19,38.25]$ & $28[20.25,38]$ & $29.5[19,39.75]$ & 0.987 \\
\hline \multicolumn{9}{|l|}{ Adjuvant chemotherapy } \\
\hline None & $16(7.0)$ & $6(6.6)$ & $10(7.4)$ & 0.329 & $10(7.1)$ & $6(8.6)$ & $4(5.7)$ & 0.288 \\
\hline Single agent regimen & $24(10.6)$ & $13(14.3)$ & $11(8.1)$ & & $15(10.7)$ & $10(14.3)$ & $5(7.1)$ & \\
\hline Multiple agents regimen & $187(82.4)$ & $72(79.1)$ & $115(84.6)$ & & $115(82.1)$ & $54(77.1)$ & $61(87.1)$ & \\
\hline
\end{tabular}

${ }^{a}$ Complications were classified according to Clavien-Dindo system

adenocarcinoma. However, considering the small sample size in each subgroup, this result shall be interpreted with caution.
For the safety evaluation of the regimens, as depicted in Table 2, after PSM, the incidence of anemia was higher in the triplet cohort, most cases required blood transfusion. 


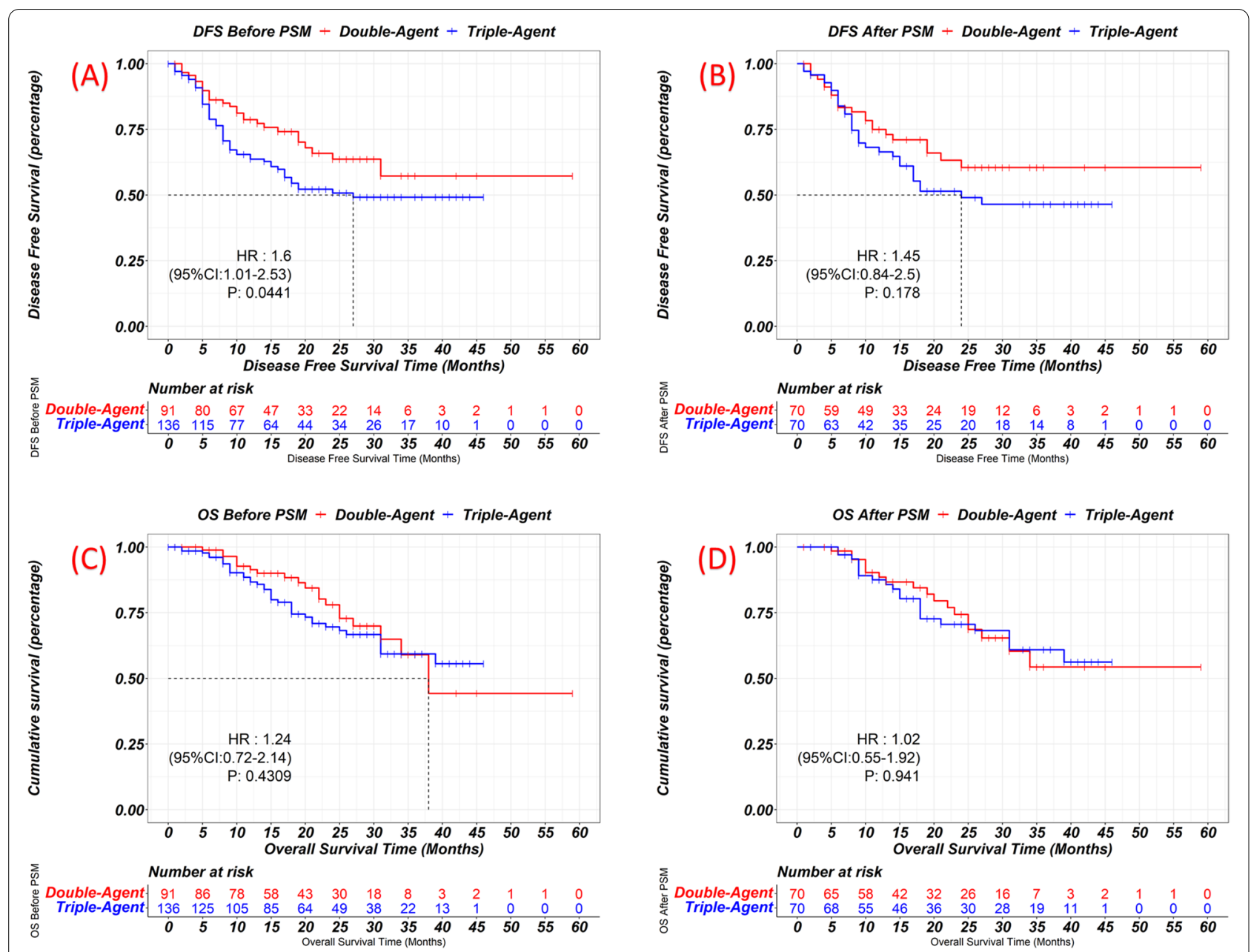

Fig. 2 The Kaplan-Meier curves showing the disease free survival of doublet and triplet cohort before (A) and after (B) PSM, and overall survival before $(\mathbf{C})$ and after (D) PSM

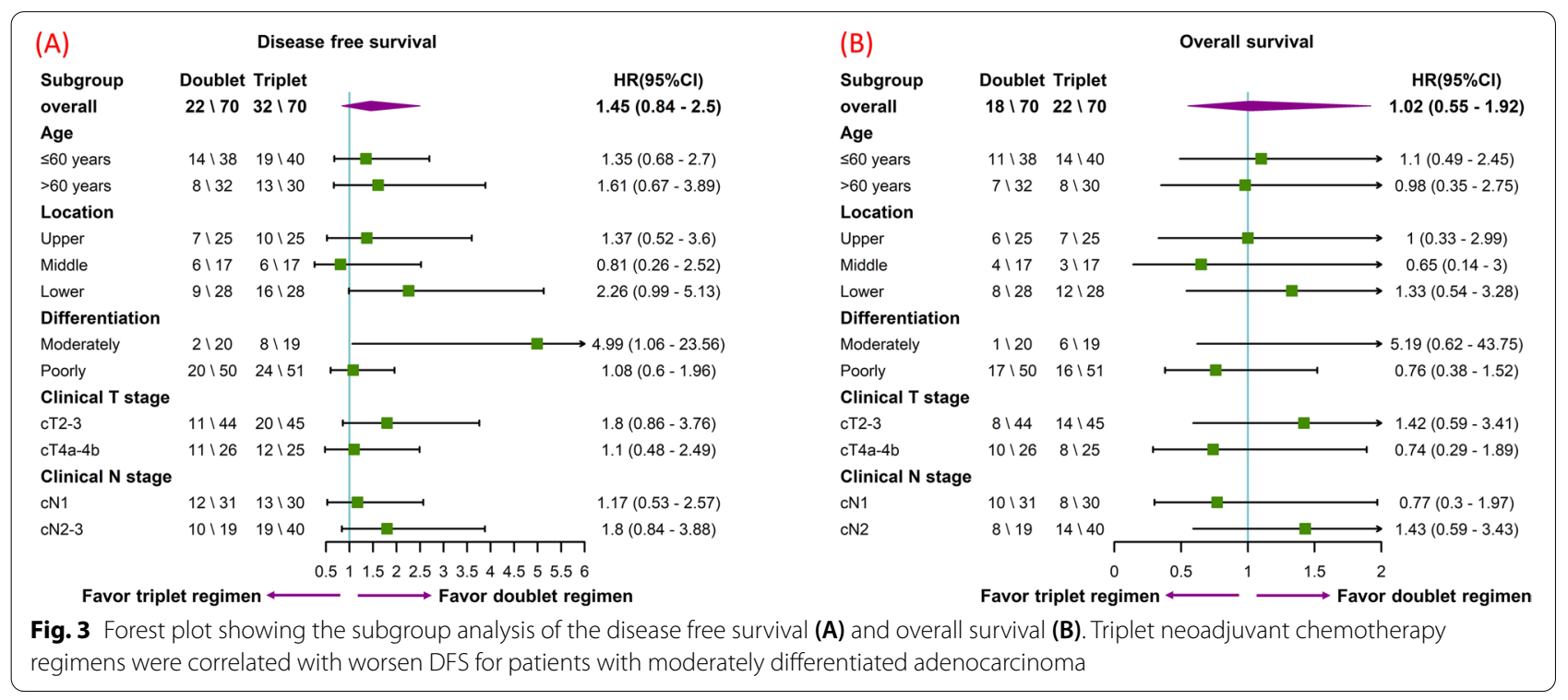


This might be related to the addition of docetaxel, as a previous study had found that docetaxel induced more severe anemia $[27,28]$. Thrombocytopenia was more frequent in the doublet cohort, which could be explained by the increased dosage of oxaliplatin per cycle, as oxaliplatin was more likely to induce thrombocytopenia [29, 30]. The incidence rate of grade $3 / 4$ toxicity that required supportive treatment was similar, though. As for perioperative safety, the Clavien-Dindo grade $2-4$ complications incidence rate was higher in the triplet cohort. The most common complication was deep abdominal infection and abscess, caused mainly by anastomotic leakage and fistula. The most likely explanation is that more intense triplet NAC regimen could induce more severe tissue edema and coagulative dysfunction, affecting the healing of the anastomosis, leading to higher risks of post-surgery complications, as proposed by previous studies [31-33]. Another possible explanation is that laparoscopic surgery could be more commonly used in the doublet cohort. Laparoscopic approaches have the benefits over open surgeries through visual magnification, better exposure, and more delicate maneuvers of organs, vessels, and nerves, all of which may contribute to a lower incidence of post-surgery complications [34].

To our knowledge, this is the first study that systematically compares the efficacy and toxicity of doublet and triplet regimens. The usage of PSM has enabled us to balance all the pre-intervention confounding factors, making the result more reliable. However, there were a few limitations to our study. Firstly, the effect of selection bias was not neglectable due to the nature of retrospective studies. Due to the fact that different oncologists might choose different regimens within the scope of the guidelines, it should be noted that the selection bias brought by doctors may be present. Secondly, the relatively small sample size had limited the reliability of the conclusion, especially for the subgroup analysis. Data of patients with the intention of surgery but eventually lost the opportunity due to the progression of the tumor during NAC were not retrievable. All the patients enrolled in the study had already received NAC and sequential surgery. However, these patients only accounted for a very small proportion, so it may not have a major impact on the result. Thirdly, the DCF regimen adopted in our study was not standardized, in which the dosage per cycle was reduced while the intervals between cycles were shortened. But the dosage density in our modified regimen remained the same as the original one, and only a few cases received this modified DCF regimen, which was unlikely to alter the final result. Fourthly, information about pre-intervention laparoscopic staging was lacking, but none of the patients enrolled in our study were found with peritoneal dissemination during the resection surgery, thus it may not alter the final conclusion. Lastly, genetic differences and other perioperative drug use such as low molecular weight heparins may also affect the prognosis, as indicated by other previous studies [35-39]. Thus, more investigations are needed to further confirm the findings.

\section{Conclusions}

Compared with doublet NAC regimens, triplet regimens may not be superior in improving tumor regression grade, R0 resection rate, and survival in patients with LAGC, and may bring a higher risk of post-surgery complications.

\begin{abstract}
Abbreviations
NAC: Neoadjuvant chemotherapy; LAGC: Locally advanced gastric cancer; PSM: Propensity score matching; ESMO: The European Society for Medical Oncology; AJCC: American Joint Committee on Cancer staging systems; NCCN: The National Comprehensive Cancer Network guideline; FLOT: Fluorouracil, leucovorin, oxaliplatin, and docetaxel; DCF: Docetaxel, cisplatin, and 5-fluorouracil; ECF: Epirubicin, cisplatin and fluorouracil; SOX: S-1/oxaliplatin; CAPOX: Capecitabine/oxaliplatin;TRG: Tumor regression grade; ECOG: Eastern Cooperative Oncology Group; FOLFOX: Fluorouracil and oxaliplatin; CT: Computerized tomography; DFS: Disease-free survival; OS: Overall survival.
\end{abstract}

\section{Consent to participate}

Informed consent were obtained from all study participants or their legal guardians prior to study by the follow-up office.

\section{Authors' contributions}

Peng JS and Lian L designed the study; Chen YH acquired, analyzed, interpreted the data, and drafted the initial manuscript. He JS and Liu D helped acquire the data and revise the manuscript. Chen XJ, Tang HJ, and Luo DD made revisions to the manuscript. Chen YH, He JS and Liu D contributed equally to this work. The author(s) read and approved the final manuscript.

\section{Funding}

This study was supported by the Research Fund of the Sixth Affiliated Hospital of Sun Yat-sen University (grant number P20200217202309876) and Nation Key Clinical Discipline.

\section{Availability of data and materials}

The datasets analysed during the current study are not publicly available due to our institution policy but are available from the corresponding author on reasonable request.

\section{Declarations}

Ethics approval and consent to participate

The study was reviewed and approved by the ethics committee of The Sixth Affiliated Hospital, Sun Yat-Sen University. This study was conducted in accordance with the 1964 Helsinki Declaration.

\section{Consent for publication}

All authors have approved the manuscript and have agreed to submit it for publication.

\section{Competing interests}

The authors declared that they have no competing interests.

\section{Author details}

${ }^{1}$ Department of Gastric Surgery, The Sixth Affiliated Hospital, Sun Yat-sen University, 26 Yuancun Erheng Road, Guangzhou 510655, China. ${ }^{2}$ Guangdong Institute of Gastroenterology, Guangdong Provincial Key Laboratory of Colorectal and Pelvic Floor Diseases, Guangzhou 510655, China. ${ }^{3}$ Department 
of Laboratory Science, The Second Affiliated Hospital, Guangzhou University of Chinese Medicine, Guangzhou 510105, China. ${ }^{4}$ Department of Medical Oncology, The Sixth Affiliated Hospital, Sun Yat-sen University, Guangzhou 510655, China.

Received: 20 August 2021 Accepted: 6 December 2021 Published online: 13 December 2021

\section{References}

1. Bray F, Ferlay J, Soerjomataram I, Siegel RL, Torre LA, Jemal A. Global cancer statistics 2018: GLOBOCAN estimates of incidence and mortality worldwide for 36 cancers in 185 countries. CA Cancer J Clin. 2018;68(6):394-424.

2. Machlowska J, Baj J, Sitarz M, Maciejewski R, Sitarz R. Gastric Cancer: Epidemiology, Risk Factors, Classification, Genomic Characteristics and Treatment Strategies. Int J Mol Sci. 2020;21(11):4012.

3. Xie Y, Shi L, He X, Luo Y. Gastrointestinal cancers in China, the USA, and Europe. Gastroenterol Rep. 2021;9(2):91-104

4. Ajani JA, D’Amico TA, Almhanna K, Bentrem DJ, Chao J, Das P, et al. Gastric Cancer, version 3.2016, NCCN clinical practice guidelines in oncology. J Natl Compr Canc Netw. 2016;14(10):1286-312.

5. Smyth EC, Verheij M, Allum W, Cunningham D, Cervantes A, Arnold D. Gastric cancer: ESMO clinical practice guidelines for diagnosis, treatment and follow-up. Ann Oncol. 2016;27(suppl 5):v38-49.

6. Wang FH, Zhang XT, Li YF, Tang L, Qu XJ, Ying JE, et al. The Chinese Society of Clinical Oncology (CSCO): Clinical guidelines for the diagnosis and treatment of gastric cancer, 2021. Cancer Commun (Lond). 2021;41(8):747-95.

7. Japanese Gastric Cancer A: Japanese gastric cancer treatment guidelines 2018 (5th edition). Gastric cancer : official journal of the International Gastric Cancer Association and the Japanese Gastric Cancer Association 2021, 24(1):1-21.

8. Al-Batran SE, Homann N, Pauligk C, Goetze TO, Meiler J, Kasper S, et al. Perioperative chemotherapy with fluorouracil plus leucovorin, oxaliplatin, and docetaxel versus fluorouracil or capecitabine plus cisplatin and epirubicin for locally advanced, resectable gastric or gastro-oesophageal junction adenocarcinoma (FLOT4): a randomised, phase 2/3 trial. Lancet (London, England). 2019;393(10184):1948-57.

9. Zhang $X$, Huang H, Wei Z, Zhu Z, Yang D, Fu H, et al. Comparison of docetaxel + Oxaliplatin + S-1 vs Oxalipatin + S-1 as neoadjuvant chemotherapy for locally advanced gastric Cancer: a propensity score matched analysis. Cancer Manag Res. 2020;12:6641-53.

10. Wang Y, Cheng X, Cui YH, Hou J, Ji Y, Sun YH, et al. Efficacy after preoperative capecitabine and oxaliplatin (XELOX) versus docetaxel, oxaliplatin and S1 (DOS) in patients with locally advanced gastric adenocarcinoma: a propensity score matching analysis. BMC Cancer. 2018;18(1):702.

11. Sah BK, Zhang B, Zhang H, Li J, Yuan F, Ma T, et al. Neoadjuvant FLOT versus SOX phase II randomized clinical trial for patients with locally advanced gastric cancer. Nat Commun. 2020;11(1):6093.

12. Amin MB, Greene FL, Edge SB, Compton CC, Gershenwald JE, Brookland RK, et al. The eighth edition AJCC Cancer staging manual: continuing to build a bridge from a population-based to a more "personalized" approach to cancer staging. CA Cancer J Clin. 2017;67(2):93-9.

13. Ryan R, Gibbons D, Hyland JM, Treanor D, White A, Mulcahy HE, et al. Pathological response following long-course neoadjuvant chemoradiotherapy for locally advanced rectal cancer. Histopathology. 2005;47(2):141-6.

14. Westerhoff $M$, Osecky $M$, Langer R. Varying practices in tumor regression grading of gastrointestinal carcinomas after neoadjuvant therapy: results of an international survey. Mod Pathol. 2020;33(4):676-89.

15. Freites-Martinez A, Santana N, Arias-Santiago S, Viera A. Using the common terminology criteria for adverse events (CTCAE - version 5.0) to evaluate the severity of adverse events of anticancer therapies. Actas dermo-sifiliograficas. 2021;112(1):90-2.

16. Lee JH, Park DJ, Kim HH, Lee HJ, Yang HK. Comparison of complications after laparoscopy-assisted distal gastrectomy and open distal gastrectomy for gastric cancer using the Clavien-Dindo classification. Surg Endosc. 2012;26(5):1287-95.

17. Duhamel A, Labreuche J, Gronnier C, Mariette C. Statistical tools for propensity score matching. Ann Surg. 2017;265(6):E79-e80.
18. Cunningham D, Allum WH, Stenning SP, Thompson JN, Van de Velde CJ, Nicolson $\mathrm{M}$, et al. Perioperative chemotherapy versus surgery alone for resectable gastroesophageal cancer. N Engl J Med. 2006;355(1):11-20.

19. Yamada Y, Higuchi K, Nishikawa K, Gotoh M, Fuse N, Sugimoto N, et al. Phase III study comparing oxaliplatin plus S-1 with cisplatin plus S-1 in chemotherapy-naïve patients with advanced gastric cancer. Ann Oncol. 2015;26(1):141-8.

20. Kim GM, Jeung HC, Rha SY, Kim HS, Jung I, Nam BH, et al. A randomized phase II trial of S-1-oxaliplatin versus capecitabine-oxaliplatin in advanced gastric cancer. Eur J Cancer (Oxford, England: 1990). 2012;48(4):518-26.

21. Satake H, Miki A, Kondo M, Kotake T, Okita Y, Hatachi Y, et al. Phase I study of neoadjuvant chemotherapy with S-1 and oxaliplatin for locally advanced gastric cancer (neo G-SOX PI). ESMO open. 2017;2(1):e000130.

22. Ji J, Shen L, Li Z, Zhang X, Liang H, Xue Y, et al. Perioperative chemotherapy of oxaliplatin combined with S-1 (SOX) versus postoperative chemotherapy of SOX or oxaliplatin with capecitabine (XELOX) in locally advanced gastric adenocarcinoma with D2 gastrectomy: a randomized phase III trial (RESOLVE trial). Ann Oncol. 2019;30:v877.

23. Wang X, Li S, Xie T, Lu Y, Guo X, Lin C. Early results of the randomized, multicenter, controlled evaluation of S-1 and oxaliplatin as neoadjuvant chemotherapy for Chinese advanced gastric cancer patients (RESONANCE trial). J Clin Oncol. 2020;38(4_suppl):280.

24. Bando E, Makuuchi R, Irino T, Tanizawa Y, Kawamura T, Terashima M. Validation of the prognostic impact of the new tumor-node-metastasis clinical staging in patients with gastric cancer. Gastric Cancer. 2019;22(1):123-9.

25. Cho H, Nakamura J, Asaumi Y, Yabusaki H, Sakon M, Takasu N, et al. Long-term survival outcomes of advanced gastric Cancer patients who achieved a pathological complete response with neoadjuvant chemotherapy: a systematic review of the literature. Ann Surg Oncol. 2015;22(3):787-92.

26. Chiou VL, Burotto M. Pseudoprogression and immune-related response in solid tumors. J Clin Oncol. 2015;33(31):3541-3.

27. Yoshida K, Kodera Y, Kochi M, Ichikawa W, Kakeji Y, Sano T, et al. Addition of docetaxel to Oral Fluoropyrimidine improves efficacy in patients with stage III gastric Cancer: interim analysis of JACCRO GC-07, a randomized controlled trial. J Clin Oncol. 2019;37(15):1296-304.

28. Migita K, Nashimoto A, Yabusaki H, Matsuki A, Aizawa M. Efficacy of neoadjuvant chemotherapy with docetaxel, cisplatin and S-1 for resectable locally advanced gastric cancer. Int J Clin Oncol. 2016;21(1):102-9.

29. Wang G, Zhao J, Song Y, Zhang W, Sun Y, Zhou A, et al. Phase II study of adjuvant chemotherapy with S1 plus oxaliplatin for Chinese patients with gastric cancer. BMC Cancer. 2018;18(1):547.

30. Lee K-W, Chung I-J, Ryu M-H, Park YI, Nam B-H, Oh H-S, et al. Multicenter phase III trial of S-1 and cisplatin versus S-1 and oxaliplatin combination chemotherapy for first-line treatment of advanced gastric cancer (SOPP trial). Gastric Cancer. 2021;24(1):156-67.

31. Claassen YHM, Hartgrink HH, Dikken JL, de Steur WO, van Sandick JW, van Grieken NCT, et al. Surgical morbidity and mortality after neoadjuvant chemotherapy in the CRITICS gastric cancer trial. Eur J Surg Oncol. 2018;44(5):613-9.

32. Hoshino N, Hida K, Sakai Y, Osada S, Idani H, Sato T, et al. Nomogram for predicting anastomotic leakage after low anterior resection for rectal cancer. Int J Color Dis. 2018;33(4):411-8.

33. Zhao DB, Zhang XJ. Thinking of the application of membrane anatomy in gastric cancer surgery after neoadjuvant therapy. Zhonghua Wei Chang Wai Ke Za Zhi. 2020;23(7):657-60.

34. Yu J, Huang C, Sun Y, Su X, Cao H, Hu J, et al. Effect of laparoscopic vs open distal gastrectomy on 3-year disease-free survival in patients with locally advanced gastric Cancer: the CLASS-01 randomized clinical trial. JAMA. 2019;321(20):1983-92.

35. Javadinia SA, Shahidsales S, Fanipakdel A, Joudi-Mashhad M, Mehramiz M, Talebian S, Maftouh M, Mardani R, Hassanian SM, Khazaei M, et al. Therapeutic potential of targeting the $W n t / \beta$-catenin pathway in the treatment of pancreatic cancer. J Cell Biochem. 2019;120(5):6833-40.

36. Fanipakdel A, Seilanian Toussi M, Rezazadeh F, Mohamadian Roshan N, Javadinia SA. Overexpression of cancer-testis antigen melanoma-associated antigen $\mathrm{A} 1$ in lung cancer: a novel biomarker for prognosis, and a possible target for immunotherapy. J Cell Physiol. 2019;234(7):12080-6. 
37. Javadinia SA, Shahidsales S, Fanipakdel A, Mostafapour A, Joudi-Mashhad $M$, Ferns GA, et al. The esophageal Cancer and the PI3K/AKT/mTOR signaling regulatory microRNAs: a novel marker for prognosis, and a possible target for immunotherapy. Curr Pharm Des. 2018;24(39):4646-51.

38. Javadinia SA, Gholami A, Joudi Mashhad M, Ferns GA, Shahidsales S, Avan A, et al. Anti-tumoral effects of low molecular weight heparins: a focus on the treatment of esophageal cancer. J Cell Physiol. 2018;233(10):6523-9.

39. Taghizadeh Kermani A, Hosseini S, Fanipakdel A, Joudi Mashhad M, Akhavan Rezayat K, Zardadi M, et al. A randomized clinical trial on the antitumoral effects of low molecular weight heparin in the treatment of esophageal cancer. J Cell Physiol. 2019;234(4):4191-9.

\section{Publisher's Note}

Springer Nature remains neutral with regard to jurisdictional claims in published maps and institutional affiliations.

- fast, convenient online submission

- thorough peer review by experienced researchers in your field

- rapid publication on acceptance

- support for research data, including large and complex data types

- gold Open Access which fosters wider collaboration and increased citations

- maximum visibility for your research: over $100 \mathrm{M}$ website views per year

At BMC, research is always in progress.

Learn more biomedcentral.com/submissions 